\title{
THE GLOBAL SOLUTION IN THE PROBLEM OF THE CRITICAL INCLINATION*
}

\author{
B. GAR FINK EL
}

Department of Astronomy, Yale University, New Haven, Conn., U.S.A.

The problem of the critical inclination in the artificial satellite theory can be regarded as a problem of resonance arising from the near-commensurability $1: 1$ between the anomalistic and the draconitic frequencies of the motion, $n_{1}$ and $n_{2}$. Let us define the main problem by the potential function

$$
V=-1 / r+J_{2} P_{2}(\sin \theta) / r^{3}+J_{4} P_{4}(\sin \theta) / r^{5},
$$

with

$$
J_{2} \ll 1, \quad J_{4}=O\left(J_{2}^{2}\right) .
$$

The classical solution of this problem, expanded in powers of the small parameter $J_{2}$, carries the critical divisor

$$
\Delta=5 \cos ^{2} i-1,
$$

as illustrated in Garfinkel (1959), Brouwer (1959), Kozai (1959), Vinti (1963), and Aksnes (1967). This divisor vanishes for the critical inclination

$$
i_{*}=\tan ^{-1} 2=63.4,
$$

which corresponds to exact commensurability $n_{1}=n_{2}$, and the solution fails in the neighborhood of $i_{*}$.

That the classical singularity at $\Delta=0$ can be removed by means of the Bohlin-type expansion (1889) in powers of $\sqrt{ } J_{2}$ is illustrated in Hori (1960) and Garfinkel (1960). They showed that the elimination of short-periodic terms from the Hamiltonian reduces the problem to the ideal resonance form as defined in Garfinkel (1966).

Although the literature of the critical inclination is quite extensive, no complete global solution of $O\left(\sqrt{ } J_{2}\right)$, valid for all inclinations, is presently available. The recent publication of a first-order solution of the ideal resonance problem has opened the way for the construction of such a theory. The present paper furnishes the algorithm for the calculation of the long-periodic and secular terms in the Delaunay variables $G, g, h$ and $l$ that are at least of the first order in $\sqrt{ } J_{2}$. The coefficients of the algorithm are expressed directly in terms of the Delaunay elements $L, G, H$ and the orbital elements $a, e, i$ of the satellite. As a check, it is shown that our global solution, valid for all inclinations, includes asymptotically the classical solution with $5 \cos ^{2} i-1$ as a divisor. The short-periodic terms, absent in our solution, can be taken from any

\footnotetext{
* Published in full in Celes. Mech. 8 (1973), 25.
} 
classical artificial satellite theory, such as Brouwer (1959). The solution is subject to the normality condition which can be written

$$
e G^{2} /\left(1+\frac{45}{4} e^{2}\right) \geqslant O\left[\mid \frac{1}{5}\left(J_{2}+J_{4} /\left.J_{2}\right|^{1 / 4}\right] .\right.
$$

The paper is divided into three parts. Part A summarizes the first-order algorithm of the ideal resonance problem, defined by the Hamiltonian

$$
F=B(y)+2 \mu^{2} A(y) \sin ^{2} x_{1}, \quad \mu \ll 1 .
$$

Here $y$ is the momentum-vector $y_{k}, x_{1}$ is the critical argument, and $x_{k}$ for $k>1$ are the ignorable coordinates, which have been eliminated from $F$ in the process of reduction to the ideal form. Part B applies the algorithm of Part A to the problem of the critical inclination. Part C, largely historical, puts the work into a proper perspective in relation to the contributions of Poincaré (1893), E. W. Brown (1931), Hori (1960), Hagihara (1961), Kozai (1961), Iszak (1962), Aoki (1963), and Jupp (1968).

\section{References}

Aksnes, K.: 1967, Astrophys. Norv. 10, 69.

Aoki, S.: 1963, Astron. J. 68, 355.

Bohlin, K. P.: 1889, 'Über eine neue Annäherungmethode in der Störungtheorie', Ak. Handl. Bihang 14 (afd. 1, Stockholm).

Brouwer, D.: 1959, Astron. J. 64, 378.

Brown, E. W. and Shook, C. A.: 1933, Planetary Theory, Dover, Ch. VIII.

Garfinkel, B.: 1959, Astron. J. 64, 353.

Garfinkel, B. : 1960, Astron. J. 65, 624.

Garfinkel, B.: 1964, Astron. J. 69, 453.

Garfinkel, B.: 1966, Astron. J. 71, 657 (Paper I).

Garfinkel, B.: 1972a, Celest. Mech. 5, 189 (Paper III).

Garfinkel, B.: 1972b, Celest. Mech. 5, 451 (Paper IV).

Garfinkel, B.: 1972c, Celest. Mech. 6, 151 (Paper V).

Garfinkel, B.: 1972d, Celest. Mech. 7, 205 (Paper VI).

Garfinkel, B., Jupp, A. H., and Williams, C. A. : 1971, Astron. J. 76, 157 (Paper II).

Hagihara, Y.: 1961, Smithsonian Contributions to Astrophys. 5, 39.

Hagihara, Y.: 1972, Celestial Mechanics, MIT Press II, Part I, p. 430.

Hori, G.: 1960, Astron. J. 65, 291.

Iszak, I.: 1962, Smithsonian Astrophys. Obs., Special Report No. 90.

Kozai, Y.: 1961, Smithsonian Contributions to Astrophys. 5, 53.

Message, P. J., Hori, G., and Garfinkel, B.: 1962, Observatory, No. 929, 168.

Poincaré, H.: 1893, Méthodes Nouvelles de la Méchanique Céleste, Volume II, p. 352, Dover Press.

Tisserand, F. F.: 1889-96, Mécanique Céleste IV, p. 444.

Vinti, J. P.: 1963, J. Res. Nat. Bur. Sd. 67B, 191.

von Zeipel, H.: 1916, Arkiv. Mat. Astron. Fys. II.

\section{DISCUSSION}

A. H. Jupp: Would you like to comment on my conjecture that your regularizing function would have to be modified if you were to formulate your solution in terms of initial values rather than in terms of the mean values?

B. Garfinkel: The elements used in my theory differ from the initial values only by quantities of the second-order. Therefore, no modification would be required in a first-order theory. As far as the second- 
order theory is concerned, some of the coefficients may have to be modified, rather than the regularizing function itself.

$T$. Inoue: Do you agree with me if I say that the perturbations depend on the choice of zeroth approximation and, therefore, if one chooses a proper intermediary orbit in the case of the ideal resonance problem one will obtain a solution free from any singularity?

B. Garfinkel: I do not agree. In my paper in Astron. J. (1964) it was shown that all classical solutions carry a singularity at the critical inclination, regardless of the choice of reference orbit or the choice of the coordinate system. 